\title{
Characteristics of muscle fiber type are predictive of skeletal muscle mass and strength in elderly men
}

Citation for published version (APA):

Verdijk, L. B., Snijders, T., Beelen, M., Savelberg, H. H., Meijer, K., Kuipers, H., \& van Loon, L. J. C. (2010). Characteristics of muscle fiber type are predictive of skeletal muscle mass and strength in elderly men. Journal of the American Geriatrics Society, 58(11), 2069-75. https://doi.org/10.1111/j.15325415.2010.03150.x

Document status and date:

Published: 01/01/2010

DOI:

10.1111/j.1532-5415.2010.03150.x

Document Version:

Publisher's PDF, also known as Version of record

Document license:

Taverne

Please check the document version of this publication:

- A submitted manuscript is the version of the article upon submission and before peer-review. There can be important differences between the submitted version and the official published version of record.

People interested in the research are advised to contact the author for the final version of the publication, or visit the DOI to the publisher's website.

- The final author version and the galley proof are versions of the publication after peer review.

- The final published version features the final layout of the paper including the volume, issue and page numbers.

Link to publication

\footnotetext{
General rights rights.

- You may freely distribute the URL identifying the publication in the public portal. please follow below link for the End User Agreement:

www.umlib.nl/taverne-license

Take down policy

If you believe that this document breaches copyright please contact us at:

repository@maastrichtuniversity.nl

providing details and we will investigate your claim.
}

Copyright and moral rights for the publications made accessible in the public portal are retained by the authors and/or other copyright owners and it is a condition of accessing publications that users recognise and abide by the legal requirements associated with these

- Users may download and print one copy of any publication from the public portal for the purpose of private study or research.

- You may not further distribute the material or use it for any profit-making activity or commercial gain

If the publication is distributed under the terms of Article $25 \mathrm{fa}$ of the Dutch Copyright Act, indicated by the "Taverne" license above, 


\title{
Characteristics of Muscle Fiber Type Are Predictive of Skeletal Muscle Mass and Strength in Elderly Men
}

\author{
Lex B. Verdijk, PhD, Tim Snijders, MSc, Milou Beelen, MD, Hans H.C.M. Savelberg, PhD, \\ Kenneth Meijer, PhD, Harm Kuipers, Prof, and Luc J.C. van Loon, PhD
}

OBJECTIVES: To investigate the relationship between skeletal muscle fiber type-specific characteristics, circulating hormone concentrations, and skeletal muscle mass and strength in older men.

DESIGN: Cross-sectional analyses.

SETTING: University research center.

PARTICIPANTS: Forty-one community dwelling elderly men $(\geq 65)$.

MEASUREMENTS: Leg strength (1-repetition maximum, $1 \mathrm{RM}$ ) and whole-body and limb muscle mass were determined, and muscle fiber type composition, cross-sectional area (CSA), myonuclear content, and satellite cell (SC) content were assessed in skeletal muscle biopsy samples. In addition, blood samples were collected to determine serum testosterone, sex hormone-binding globulin, insulinlike growth factor (IGF)-1, and IGF binding protein-3 concentrations.

RESULTS: Muscle mass correlated with muscle strength $(0.41 \leq$ correlation coefficient $(r) \leq 0.72 ; P<.01)$. Muscle fiber CSA, myonuclear content, and SC content were significantly lower in type II than in type I muscle fibers. Myonuclear and SC content were positively correlated with muscle fiber CSA. Furthermore, greater muscle fiber CSA (type I and II) was associated with greater thigh muscle area and muscle strength $(0.30 \leq r \leq 0.45 ; P<.05)$. Testosterone concentration was positively correlated with muscle mass and muscle fiber CSA. Regression analysis showed that SC content, myonuclear content, and testosterone concentration are predictive of muscle fiber CSA. Furthermore, muscle mass and type II muscle fiber CSA are predictive of muscle strength.

CONCLUSION: Skeletal muscle mass and strength in elderly men are positively correlated with muscle fiber type-specific CSA, myonuclear content, and SC content.

From the Department of Human Movement Sciences, NUTRIM School for Nutrition, Toxicology and Metabolism, Maastricht University Medical Center, Maastricht, the Netherlands.

Address correspondence to Lex Verdijk, Department of Human Movement Sciences, Faculty of Health, Medicine and Life Sciences, Maastricht University Medical Centre+, P.O. Box 616, 6200 MD Maastricht, the Netherlands.

E-mail: Lex.Verdijk@bw.unimaas.nl

DOI: $10.1111 /$ j.1532-5415.2010.03150.x
These findings support the assumption that a decline in SC content plays an important role in age-related decline in muscle mass and strength. J Am Geriatr Soc 58:2069-2075, 2010.

Key words: sarcopenia; muscle fiber size; satellite cells; aging; hypertrophy

ging is associated with the gradual loss of skeletal
muscle mass and function, termed sarcopenia. The
prevalence of sarcopenia is approximately $25 \%$ after the
age of 60 , increasing to up to approximately $50 \%$ in people
aged 80 and older. ${ }^{1-3}$ The latter is in accordance with the
progressive nature of the loss of muscle mass ${ }^{4-8}$ and muscle
strength ${ }^{6,9}$ with aging. The etiology of sarcopenia is gen-
erally studied in a cross-sectional manner within a large
population of individuals ranging in age from 20 to 80 ,
although few data are available on the determinants of
muscle mass and strength within an elderly population.
Some studies have reported that muscle mass and strength
continue to decline after the age of $65.10,11$ This seems
consistent with the greater prevalence of sarcopenia in peo-
ple aged 80 and older than in those aged 60 and older ${ }^{1-3}$
and agrees with longitudinal changes reported previ-
ously. ${ }^{2,13}$ Nevertheless, there is a large variability in mus-
cle mass and strength within the elderly population that is
not necessarily related to age per se. ${ }^{14}$ Therefore, studying
determinants of muscle mass within the elderly population
(independent of age) could provide better insight into the
key etiological factors of sarcopenia.
Leg skeletal muscle mass seems to be the main deter-
minant of leg muscle strength, independent of age. ${ }^{15,16}$ The
loss of leg muscle mass in older adults has been attributed to
a decline in the number of muscle fibers and, more specifi-
cally, to type II muscle fiber atrophy. ${ }^{8,17,18}$ These findings
were recently extended by showing a type II muscle fiber-
specific decline in satellite cell (SC) content in older mus-
cle. ${ }^{19}$ It has been suggested that such a decline in muscle 
fiber SC content plays an important role in muscle fiber atrophy ${ }^{20,21}$ and the subsequent loss of muscle mass with aging. It could be speculated that SC content, muscle fiber size, and muscle mass are closely linked, independent of age. Therefore, it was hypothesized that, in an elderly population, muscle fiber size and SC content are correlated and are predictive of whole-body and limb skeletal muscle mass and strength.

There have been ample suggestions that age-related changes in hormonal profile can strongly modulate skeletal muscle mass and strength in older adults. ${ }^{22}$ In accordance, significant correlations have been reported between lean mass and muscle strength and serum testosterone and insulinlike growth factor (IGF)-1 concentrations in elderly men. $^{2,14,23}$ Furthermore, dose-dependent increases in lean mass, strength, muscle fiber size, and SC content have been reported after testosterone supplementation in younger and older men, ${ }^{24,25}$ which is indicative of a role for anabolic hormones in regulating SC-induced muscle fiber hypertrophy. The goal of the present study was to extend previous findings ${ }^{24,25}$ by investigating the relationship between baseline serum hormone profiles and muscle characteristics. In line with potential hormonal regulation of SC, it was hypothesized that baseline hormone concentrations (particularly testosterone) are predictive not only of skeletal muscle mass and strength, but also of SC content and muscle fiber size.

The present study aimed to investigate the relationship between skeletal muscle fiber type-specific characteristics, circulating hormone concentrations, and skeletal muscle mass and strength in older men. Skeletal muscle characteristics were assessed at the whole-body, limb, and muscle fiber level, and circulating hormone concentrations were determined in a group of community-dwelling older men.

\section{METHODS}

\section{Participants}

Forty-one older men (65-86) were included in the present study. All participants participated in different studies that are part of a greater project investigating the clinical benefits of exercise and nutritional intervention in older men for which participants were recruited through advertisements in local newspapers. Medical history of all participants was evaluated, and an oral glucose tolerance test (OGTT) and resting electrocardiogram were performed. Participants with (silent) cardiac or peripheral vascular disease, orthopedic limitations, or type 2 diabetes mellitus ${ }^{26}$ were excluded. All participants were living independently and had no history of participating in any structured exercise training program for at least 5 years. Furthermore, participants reported no problems in normal activities of daily living (e.g., walking, climbing stairs, rising from a chair) and did not need any assistive equipment (e.g., using a cane) while walking. Participants taking antihypertensive medications $(n=13)$, cholesterol- or lipid-lowering medications $(\mathrm{n}=8)$, medications for prostate or urinary problems $(n=8)$, or no medications $(n=20)$ were included in the study. Individuals with more-severe medical problems (treatment by medical specialist more than twice a year) were excluded. Participants from the larger cohort $(\mathrm{n}=59)$ were included in the present analysis when a muscle biopsy was available, with at least 75 type I and 75 type II muscle fibers being analyzed, which is required to reliably assess myonuclear and SC content. ${ }^{27}$ No differences were observed between the participants included in the present study and the larger cohort regarding age, body composition, and muscle mass and strength (Table 1). All participants were informed of the nature and possible risks of the experimental procedures before written informed consent was obtained. The Medical Ethics Committee of the Maastricht University Medical Center approved the study.

\section{Study Design}

After inclusion in this study, all participants underwent the same series of measurements (outlined below) to determine muscle characteristics at the whole-body, limb, and myocellular level. In addition, a single fasting blood sample was collected from every participant between 8:00 and 9:00 a.m. to assess hormonal profile. On all test days (muscle biopsy and blood sampling and dual energy X-ray absorptiometry (DXA) and computed tomography (CT) scanning), participants arrived at the laboratory by car or public transportation at 8:00 a.m. and in an overnight fasting state. In addition, participants were asked to maintain their normal physical activity pattern in the 3 days before muscle biopsy collection (no unaccustomed strenuous physical exercise or labor) to prevent any confounding transient perturbations in baseline SC content.

\section{Strength Assessment}

Maximum strength was assessed according to 1-repetition maximum (1RM) strength tests on leg press and leg extension machines (Technogym, Rotterdam, the Netherlands). During a familiarization trial, proper lifting technique was demonstrated and practiced. Maximum strength was estimated using the multiple-repetition testing procedure. ${ }^{28}$ In an additional session, at least 1 week before muscle biopsy collection, each participant's 1RM was determined as described previously; ${ }^{29} 1 \mathrm{RM}$ as determined from the second session was always the largest value recorded.

\begin{tabular}{lcc}
\hline Table 1. Subject Characteristics & \\
\hline & \multicolumn{2}{c}{ Mean \pm Standard Deviation (range) } \\
\cline { 2 - 3 } & $\begin{array}{c}\text { Present Study } \\
\text { Characteristic }\end{array}$ & $\begin{array}{c}\text { Total Cohort } \\
(\mathbf{n}=\mathbf{5 9})\end{array}$ \\
\hline Age & $72 \pm 5(65-86)$ & $72 \pm 5(65-86)$ \\
Body mass, kg & $80.3 \pm 11.8(63.1-102.2)$ & $79.8 \pm 11.1(63.0-102.2)$ \\
Height, $\mathrm{m}$ & $1.73 \pm 0.06(1.61-1.86)$ & $1.72 \pm 0.05(1.61-1.86)$ \\
Body mass index, & $27.0 \pm 3.5(21.6-34.6)$ & $26.8 \pm 3.2(21.6-34.6)$ \\
kg/m ${ }^{2}$ & & \\
Fasting glucose, & $5.7 \pm 0.6(4.5-6.9)$ & $5.7 \pm 0.6(4.5-6.9)$ \\
mmol/L & & \\
Body fat, \% & $25.4 \pm 6.8(10.6-39.8)$ & $25.3 \pm 6.5(10.6-39.8)$ \\
Lean mass, kg & $56.1 \pm 5.3(49.0-71.1)$ & $55.9 \pm 4.9(48.8-71.1)$ \\
1RM leg extension, kg & $84 \pm 13(50-110)$ & $85 \pm 12(50-110)$ \\
1RM leg press, kg & $170 \pm 28(105-225)$ & $170 \pm 27(105-225)$ \\
\hline
\end{tabular}

No differences were observed between the subjects included in the present study, and the total cohort from which this group was selected.

$1 \mathrm{RM}=1$-repetition maximum. 


\section{Thigh Muscle Cross-Sectional Area}

Anatomical cross-sectional area (CSA) of the thigh muscles was assessed using CT scanning (IDT 8000, Philips Medical Systems, the Netherlands), as described previously. ${ }^{30}$ Scans were performed at the mid-thigh level, and images were loaded onto a personal computer using AGFA IMPAX imaging software, version 5.2 (AGFA Healthcare, Brussels, Belgium). Muscle area of the right leg was selected between -29 and +150 Hounsfield units, ${ }^{31}$ after which the quadriceps muscle was selected using manual tracing. Total thigh and quadriceps muscle area were calculated using Lucia 4.81 software (Nikon Instruments Europe, Badhoevedorp, the Netherlands). Two investigators blinded to participant coding performed all analyses; intraclass correlation coefficients for inter- and intrainvestigator reliability were 0.997 and 0.998 , respectively.

\section{Body Composition}

Directly after CT scanning, body composition and bone mineral content were measured using DXA (Lunar Prodigy Advance, GE Healthcare, Madison, WI). The system's software package (enCORE 2005, version 9.15.00) was used to determine whole-body and regional lean and fat mass. DXA scans were performed in a fasting state after participants had voided. The coefficient of variation $(\mathrm{CV})$ for repetitive scans $(\mathrm{n}=4,2$ weeks apart) were $0.4 \%, 1.0 \%$, and $1.1 \%$ for whole-body lean mass, fat mass, and leg lean mass, respectively. Appendicular skeletal muscle mass (ASM) was calculated as the sum of lean mass of the arms and legs. ${ }^{1}$ Body mass was measured to the nearest $0.1 \mathrm{~kg}$ using an electronic balance scale, and height was measured to the nearest $0.1 \mathrm{~cm}$ using a wall-mounted stadiometer. To control for the potential confounding effect of body size, all body composition measures were adjusted for knee height. ${ }^{32}$ Knee height was chosen instead of full height, because age-related disorders affecting the spine (e.g., osteoporosis) can confound the latter. ${ }^{33}$

\section{Blood Samples}

Blood samples were collected in ethylenediaminetetraacetic acid-containing tubes and serum tubes. After centrifugation, aliquots of plasma and serum were frozen in liquid nitrogen and stored at $-80^{\circ} \mathrm{C}$. Plasma glucose concentrations were analyzed using a COBAS FARA analyzer (Uni Kit III, Roche, Basel, Switzerland). Total serum testosterone and sex hormone-binding globulin (SHBG) concentrations were measured using reagents from Roche Diagnostics (Mannheim, Germany), and assays were run on a Modular Analytics E170 analyzer (Hitachi Data Systems, Santa Clara, CA). The intraassay CVs are $2.7 \%$ to $1.8 \%$ and $1.1 \%$ to $1.7 \%$ for low to high concentrations of testosterone and SHBG, respectively. Bioavailable testosterone was calculated as non-SHBG-bound testosterone using a formula described and validated previously. ${ }^{34}$ IGF-1 was analyzed on a Liaison system (DiaSorin, Brussels, Belgium) with reagents from DiaSorin. Intraassay CVs are $2.4 \%$ to $4.4 \%$ for high and low concentrations, respectively. IGF binding protein (BP)-3 was analyzed using a commercially available test kit (Biosource Europe, Nivelles, Belgium) on a Tecan/GENios analyzer (Tecan Group Ltd., Männedorf, Switzerland), with intraassay CVs of $4 \%$ to $8 \%$.

\section{Muscle Biopsy Sampling}

Muscle biopsy samples were collected from the right leg in the morning after an overnight fast. After local anesthesia, percutaneous needle biopsies $(50-80 \mathrm{mg})$ were taken from the vastus lateralis muscle, approximately $15 \mathrm{~cm}$ above the patella. ${ }^{35}$ Any visible nonmuscle tissue was removed immediately, and biopsy samples were embedded in Tissue-Tek (Sakura Finetek, Zoeterwoude, the Netherlands), frozen in liquid nitrogen-cooled isopentane, and stored at $-80^{\circ} \mathrm{C}$.

\section{Immunohistochemistry}

From all biopsies, 5 - $\mu$ m-thick cryosections were cut at $-20^{\circ} \mathrm{C}$. Samples from two participants were mounted together on uncoated glass slides. Care was taken to align the samples properly for cross-sectional fiber analyses. Serial cross-sections were stained for muscle fiber typing and myocellular SC content. Details of the analytical procedures have been described previously. ${ }^{30}$ In short, muscle fiber typing (type I vs II) was determined based on myosin heavy chain staining, and a CD56 antibody was used to determine SC content. Laminin was used to visualize the basement membrane, and nuclei were stained with 4',6-diamidino2-phenylindole (DAPI). After staining, all images were digitally captured using fluorescence microscopy (Nikon Instruments Europe). Image processing and quantitative analyses were done using the Lucia 4.81 software package. An investigator blinded to participant coding performed all image recordings and analyses. Within each image, the number of fibers, the mean fiber CSA, the number of myonuclei per fiber, and the myonuclear domain (fiber CSA/ number of myonuclei) were measured for the type I and type II muscle fibers separately. For the SC slides, fiber typing was determined by matching the serial fiber typing slides. SCs were determined at the periphery of each fiber and stained positive for deoxyribonucleic acid (DAPI) and CD56. The number of SCs per muscle fiber and the percentage of SC [number of SCs/(number of SC+number of myonuclei) $\times 100$ ] were determined for the type I and II muscle fibers separately. A mean total of $338 \pm 152$ muscle fibers (178 \pm 90 type I and $160 \pm 77$ type II muscle fibers) were analyzed for each participant. To determine interobserver reliability for the number of SCs per fiber, two investigators blinded to participant coding analyzed five randomly chosen biopsy specimens; the CV was $1.5 \%$. Furthermore, the agreement in determination of the fiber type to which SCs belong was shown to be $99 \%$.

\section{Statistics}

Sample size was calculated based on previous findings; 9,36 20 to 39 participants were needed to detect significant correlations between muscle fiber CSA, muscle mass, and muscle strength (with power $=0.80$ and $\alpha=0.05$ ). Kolmogorov-Smirnov tests showed that age was the only variable that was not normally distributed. To determine the relationship between age and all other variables (measured using DXA, CT, muscle biopsy, and blood sample analyses), Spearman rank correlation coefficients $(r)$ were calculated. The relationship between the various muscle characteristics and between muscle characteristics and hormonal profiles were determined by calculating bivariate 
Table 2. Characteristics of Lean Body Mass and Their Correlations with Age and Muscle Strength

\begin{tabular}{|c|c|c|c|c|c|c|c|}
\hline Characteristic & Mean \pm SD & $\begin{array}{c}r \text { Age, } \\
\text { Mean } \pm \text { SEE }\end{array}$ & $P$-Value & $\begin{array}{c}r \text { 1RM-LE, } \\
\text { Mean } \pm \text { SEE }\end{array}$ & P-Value & $\begin{array}{c}r \text { 1RM-LP, } \\
\text { Mean } \pm \text { SEE }\end{array}$ & $P$-Value \\
\hline Whole-body lean mass, $\mathrm{kg}$ & $56.1 \pm 5.3$ & $-0.24 \pm 0.16$ & .07 & $0.41 \pm 0.15$ & .005 & $0.48 \pm 0.14$ & .001 \\
\hline Arm lean mass, $\mathrm{kg}$ & $6.5 \pm 0.8$ & $-0.26 \pm 0.16$ & .05 & $0.54 \pm 0.13$ & $<.001$ & $0.45 \pm 0.14$ & .002 \\
\hline Appendicular skeletal muscle mass, $\mathrm{kg}^{*}$ & $24.3 \pm 2.5$ & $-0.36 \pm 0.15$ & .01 & $0.51 \pm 0.14$ & $<.001$ & $0.57 \pm 0.13$ & $<.001$ \\
\hline Quadriceps muscle CSA, $\mathrm{cm}^{2}$ & $73.0 \pm 11.3$ & $-0.50 \pm 0.13$ & $<.001$ & $0.72 \pm 0.11$ & $<.001$ & $0.67 \pm 0.12$ & $<.001$ \\
\hline
\end{tabular}

Spearman rank (for age) and Pearson (for one-repetition maximum (1RM) for leg extension (LE) and leg press (LP)) correlation coefficients $(r)$.

* Sum of lean mass in arms and legs.

$\mathrm{SEE}=$ standard error of the estimate; $\mathrm{CSA}=$ cross-sectional area.

Pearson correlation coefficients. In addition, partial correlations (adjusting for the effect of age) were calculated, eliminating age as a contributing factor to the correlation. Likewise, the correlation between age and muscle strength was adjusted for the effect of quadriceps CSA. Differences between the various correlation coefficients were tested for statistical significance. ${ }^{37}$ Differences between type I and II muscle fibers were analyzed using paired-samples $t$-tests. Forward linear regression modeling was used to identify the main predictors for muscle mass and strength (quadriceps and thigh CSA, and 1RM leg press and leg extension) and muscle fiber size. Based on the results of the correlation analysis, a number of independent variables were included as potential predictors. Because age was not normally distributed, this variable was fit as a categorical variable $(<70$, $70-74$, and $\geq 75$ ). All analyses were performed using SPSS version 15.0 (SPSS, Inc., Chicago, IL). An $\alpha$-level of .05 was used to determine statistical significance. Differences between type I and II muscle fibers were determined using two-sided tests. Correlation coefficients were determined using one-sided tests because specific directions were expected for the correlations studied. All data are presented as means \pm standard deviations.

\section{RESULTS}

Participant characteristics are provided in Table 1. The mean age of the participants was $72 \pm 5$ (range 65-86). Basal blood glucose concentrations, glycosylated hemoglobin levels, and oral glucose tolerance were within the normal range for healthy older men.

\section{Age-Related Changes}

Age did not correlate with whole-body lean mass, although there was a significant negative correlation between age and regional measures of muscle mass, with the strongest correlations observed between age and total thigh and quadriceps muscle CSA (Table 2). In addition, for the leg press and leg extension exercises, muscle strength was negatively correlated with age $(r=-0.38$ and $-0.37, P=.008$ and .009 , respectively). When adjusted for the effect of quadriceps muscle CSA, the correlation between age and muscle strength disappeared (leg press: $r=-0.10, P=.52$, leg extension: $r=-0.14 P=.41$ ).

At the myocellular level, type II muscle fiber CSA was shown to be smaller than type I muscle fiber CSA (Table 3).
In addition, myonuclear and SC content were lower in the type II than the type I muscle fibers. Whereas the percentage of type I and II muscle fiber was similar, the percentage of type II muscle fiber area was smaller than of type I muscle fiber area. In contrast to type I muscle fiber CSA, type II muscle fiber CSA correlated significantly with age $(r=-0.23 ; P=.04)$.

\section{Whole-Body, Regional, and Myocellular Characteristics}

All measures of lean mass (whole-body and regional lean mass) and thigh and quadriceps CSA showed positive correlations with each other and with leg extension and leg press strength (Table 2). Correlations between quadriceps CSA and leg extension strength $(r=0.72)$ and between thigh CSA and leg press strength $(r=0.72)$ were most pronounced $(P<.001)$. Partial correlation coefficients showed that the relationships between muscle mass and strength did not change when adjusted for age.

For the type I and II muscle fibers, greater muscle fiber CSA was associated with more myonuclei per fiber, a greater myonuclear domain, and more SCs per muscle fiber (Table 4). In addition, the number of myonuclei per muscle fiber correlated positively with the number of SCs per muscle fiber ( $r=0.40$ for type I and II muscle fibers; $P=.006$ ).

Type I and II muscle fiber CSA showed a positive correlation with leg extension strength (Figure 1) but not with leg press strength. Furthermore, greater muscle fiber CSA

\begin{tabular}{lcc}
\hline \multicolumn{3}{c}{ Table 3. Muscle Fiber Type Characteristics } \\
\hline & \multicolumn{2}{c}{ Mean \pm Standard Deviation } \\
\cline { 2 - 3 } Characteristic & Type I & Type II \\
\hline Fiber, \% & $52 \pm 13$ & $48 \pm 13$ \\
CSA, $\mu \mathrm{m}^{2}$ & $6,460 \pm 1,662$ & $5,276 \pm 1,402^{*}$ \\
CSA\% & $57 \pm 14$ & $43 \pm 14^{*}$ \\
Nuclei/fiber & $3.3 \pm 0.8$ & $2.7 \pm 0.7^{*}$ \\
Nuclear domain, $\mu \mathrm{m}^{2}$ & $2,036 \pm 75$ & $1,970 \pm 79$ \\
SCs/fiber & $0.087 \pm 0.031$ & $0.050 \pm 0.016^{*}$ \\
SC\% & $2.7 \pm 0.9$ & $1.8 \pm 0.6^{*}$ \\
\hline
\end{tabular}

* Significantly different from type I muscle fibers.

CSA $=$ cross-sectional area; $\mathrm{CSA} \%=$ percentage of total area occupied per fiber type; $\mathrm{SC}=$ satellite cell; SC\% $=$ percentage of SCs (number of SCs/ (number of SCs + number of myonuclei) $\times 100 \%$ ). 
Table 4. Correlations Between Skeletal Muscle Characteristics and Muscle Fiber Cross-Sectional Area (CSA)

Pearson Correlation

Coefficient \pm Standard Error of the Estimate, $P$-Value

\begin{tabular}{lll}
\cline { 2 - 3 } \multicolumn{1}{c}{ Characteristic } & \multicolumn{1}{c}{ Type I CSA } & \multicolumn{1}{c}{ Type II CSA } \\
\hline Nuclei/fiber & $0.56 \pm 0.13,<.001$ & $0.56 \pm 0.13,<.001$ \\
Nuclear domain & $0.41 \pm 0.15, .005$ & $0.50 \pm 0.14, .001$ \\
Satellite cells/fiber & $0.55 \pm 0.13,<.001$ & $0.50 \pm 0.14,<.001$ \\
Quadriceps muscle CSA & $0.33 \pm 0.15, .02$ & $0.39 \pm 0.15, .006$ \\
Thigh CSA & $0.30 \pm 0.15, .03$ & $0.39 \pm 0.15, .006$ \\
One-repetition maximum & & \\
Leg extension & $0.32 \pm 0.15, .02$ & $0.45 \pm 0.14, .002$ \\
Leg press & $0.07 \pm 0.16, .33$ & $0.14 \pm 0.16, .19$ \\
\hline
\end{tabular}

was associated with greater quadriceps CSA and greater thigh CSA (Table 4). Adjusting for age did not modulate any of the correlations.

\section{Hormonal Profiles and Muscle Characteristics}

Average serum concentrations of total testosterone, bioavailable testosterone, SHBG, IGF-I, and IGF-BP3 were $16.1 \pm 4.8 \mathrm{nmol} / \mathrm{L}, 5.8 \pm 1.6 \mathrm{nmol} / \mathrm{L}, 52.3 \pm 12.7 \mathrm{nmol} / \mathrm{L}$, $15.1 \pm 4.8 \mathrm{nmol} / \mathrm{L}$, and $76.1 \pm 11.8 \mathrm{nmol} / \mathrm{L}$, respectively. Higher total testosterone concentrations were associated with higher serum SHBG concentrations $(r=0.52$, $P<.001)$. Bioavailable testosterone concentrations correlated positively with IGF-1 $(r=0.31, P=.02)$. In addition, a positive correlation was observed between IGF-1 and IGF-BP3 $(r=0.46, P=.001)$.

Higher levels of bioavailable testosterone were associated with greater ASM $(r=0.26 ; P=.04)$ and greater muscle mass measured using CT $(r=0.27 ; P=.04)$. Whereas type II muscle fiber CSA was greater with higher concentrations of bioavailable testosterone expressed as a percentage of total testosterone $(r=0.30 ; P=.03)$, this correlation was not significant for type I muscle fibers $(r=0.23 ; P=.07)$. Furthermore, higher bioavailable testosterone levels were associated with a larger percentage of fiber area occupied by type II muscle fibers $(r=0.31 ; P=.02)$. No positive correlations were observed for type I and II muscle fiber myonuclear or SC content with any of the hormones measured.

\section{Linear Regression Analysis}

Based on the outcome of the correlation analysis, potential predictors of muscle mass and strength were analyzed in a regression model. For muscle strength, the independent variables were age, quadriceps and thigh CSA, bioavailable testosterone concentration, and type I and II muscle fiber CSA. For muscle mass, the independent variables were age, bioavailable testosterone concentration, and type I and II muscle fiber CSA. For muscle fiber type I and II CSA, the independent variables were age, bioavailable testosterone concentration, and myonuclear and SC content (type I and II, respectively).

Total thigh CSA alone was shown to predict 1RM leg press (coefficient of determination $\left(R^{2}\right)=0.52$ ), and quad- riceps CSA and type II muscle fiber CSA predicted 1RM leg extension $\left(R^{2}=0.54\right)$. Age and type II muscle fiber CSA significantly predicted total thigh $\left(R^{2}=0.28\right)$ and quadriceps CSA $\left(R^{2}=0.32\right)$. Finally, the number of myonuclei per fiber, the number of SCs per fiber, and bioavailable testosterone concentration significantly predicted type I $\left(R^{2}=\right.$ $0.49)$ and type II $\left(R^{2}=0.48\right)$ muscle fiber CSA.

\section{DISCUSSION}

The present study shows that muscle fiber type characteristics, bioavailable testosterone concentration, and muscle mass are closely correlated, providing important predictors of skeletal muscle mass and strength in older men. The findings indicate that SC content and muscle fiber CSA are tightly coupled, concurrent with the assumption that a decline in SC content plays an important role in type II muscle fiber specific atrophy and, as such, in the loss of skeletal muscle mass and strength in older men.

The present study shows that skeletal muscle mass correlates strongly with muscle strength in older men. Although longitudinal changes were not studied, this finding supports the concept that the loss of muscle mass is associated with muscle weakness, resulting in functional impairment in older adults. ${ }^{16,38}$ Previous studies with participants selected over a wide age range $(18-80)$ reported significant correlations between strength and muscle fiber size. ${ }^{9,36}$ The current study extends these findings by showing that, independent of age, smaller type I and II muscle fiber size is associated with a smaller quadriceps CSA and lower leg strength in an older male population. The correlation between muscle fiber size and muscle strength tended to be stronger for type II than type I muscle fibers (Figure 1). Although speculative, this might be partly attributed to the observation that type II muscle fibers can generate greater specific tension, explaining their importance for explosive force production. ${ }^{39}$ As such, specific type II muscle fiber atrophy with aging contributes to the development of muscle weakness from a quantitative (loss

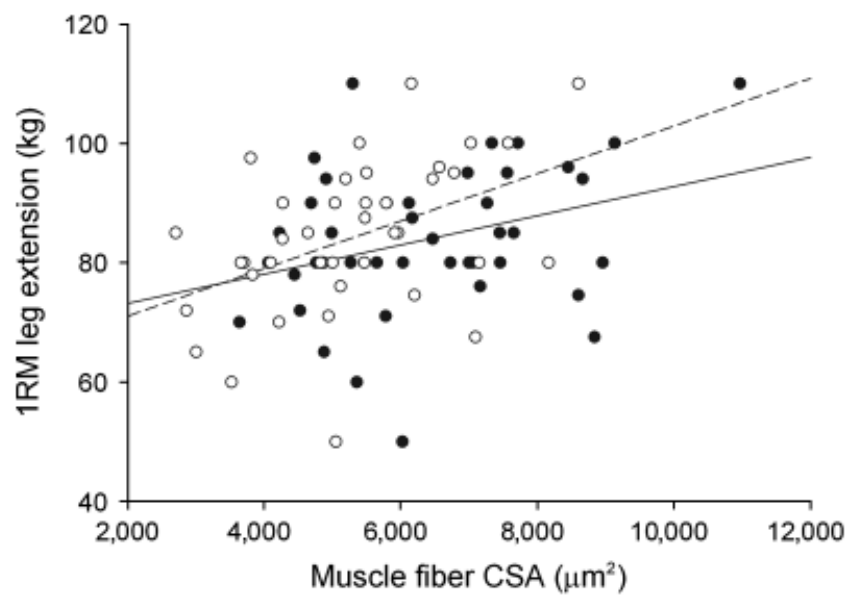

Figure 1. Scatter plot for the correlation of type I (filled circles) and type II (open circles) muscle fiber cross-sectional area (CSA) with one-repetition maximum (1RM) leg extension strength. Lines represent the fitted regression. Pearson correlation coefficients $(r)$ tended to be stronger $(P=.06)$ for type II (dashed line, $r=0.45, P=.002$ ) than type I muscle fibers (solid line, $r=0.33$, $P=.02)$. 
of muscle fiber area) and a qualitative (relative increase in type I muscle fiber area) perspective. The finding that quadriceps muscle fiber size was significantly correlated with leg extension but not leg press strength (Table 4) is probably related to the fact that leg extension represents isolated quadriceps and knee extension strength, whereas leg press represents combined hip, knee, and ankle extension strength. ${ }^{29}$ As such, other factors, such as the ability to coordinate force production in different muscles and joints, may have obscured the potential correlation between muscle fiber size and leg press strength.

The correlation between age and muscle strength disappeared when corrected for muscle CSA. In accordance, regression analysis showed that muscle mass and type II muscle fiber size are the main predictors of muscle strength (explaining $>50 \%$ of the variance). In general, muscle mass and quality determine muscle strength. Because age was not shown to contribute significantly to the variability in strength, it seems that age does not affect muscle quality per se. Other factors, such as type II muscle fiber atrophy, greater intramuscular lipid or connective tissue content, ${ }^{40,41}$ impaired neural function, ${ }^{4}$ and a lower physical activity level ${ }^{4,14}$ have been associated with poorer muscle quality and, as such, lower muscle strength. Because these aspects were not specifically addressed in the present study, their relative importance for muscle strength remains to be determined.

Type II muscle fiber CSA was shown to be smaller than type I muscle fiber CSA (Table 3). In addition, myonuclear and SC content were lower in type II than type I muscle fibers, which is consistent with previous findings. $8,17,19,30,42$ Type I and type II muscle fiber SC and myonuclear content were positively correlated with muscle fiber CSA, consistent with the myonuclear domain theory. ${ }^{43-45}$ It has been proposed that changes in SC content play an important role in age-related skeletal muscle atrophy ${ }^{19-21,46-48}$ and exerciseinduced muscle hypertrophy. ${ }^{30,43}$ Therefore, it was speculated that skeletal muscle SC content acts as an important regulator of muscle fiber size, as the outcome of the regression analysis supports, showing that SC and myonuclear content can be predictive of muscle fiber size. As such, the muscle fiber type-specific decline in SC content observed with aging seems to represent an important factor in the age-related loss of muscle mass and strength.

Consistent with previous findings, ${ }^{2,23}$ a positive correlation was found between bioavailable testosterone levels and muscle mass (CT, DXA) and muscle fiber size. Together with SC and myonuclear content, bioavailable testosterone was also shown to be predictive of muscle fiber size, although despite previous findings, ${ }^{25}$ no correlation was seen between testosterone and myonuclear or SC content. Although this might imply that the primary action of testosterone is not directed toward SCs, more research is warranted to address this question. Is has been suggested that local and systemic IGF-1 play a role in regulating the SC cycle ${ }^{20,49}$ although no correlations were observed between IGF-1 concentrations and SC content. Moreover, in contrast to previous studies, ${ }^{14,23}$ no correlations were observed between IGF-1 and various measures of muscle mass. The small variability in IGF-1 and muscle mass in the participant population included in the present study might explain these findings. Alternatively, it might be that muscle-specific IGF-1 is a stronger predictor of muscle mass and myogenic potential, and future research should further delineate the specific roles of muscle-specific and systemic IGF-1 in regulating skeletal muscle mass. ${ }^{49}$

The present cross-sectional analysis does not allow any direct causal relationships to be determined. Age-related changes in muscle characteristics (e.g., muscle fiber atrophy) generally represent slowly progressing processes, taking 20 to 30 years to become apparent. ${ }^{8}$ As such, longitudinal studies are difficult to assess because of methodological problems regarding follow-up duration and high dropout rates. In the present study, whether the known agerelated changes in muscle fiber characteristics can explain the large variance in muscle mass and strength in a population of community-dwelling elderly men was therefore assessed. This is the first study to show predictive associations between SC content, muscle fiber size, and skeletal muscle mass and strength in elderly men.

The sample size of the present study and the inclusion of only relatively healthy elderly men may limit its potential for generalization to other subpopulations such as women or frail older men. Nonetheless, combined with the findings from recent intervention studies ${ }^{25,30}$ and in vivo animal experiments, ${ }^{21,43,46}$ the current data indicate that SCs play an important role in the age-related loss of muscle mass and function.

In conclusion, muscle mass and muscle fiber size are predictive of skeletal muscle strength in elderly men. Furthermore, SC content, myonuclear content, and bioavailable testosterone concentration seem to play important roles in regulating muscle fiber size and, as such, skeletal muscle mass and strength in older men. These findings provide further support to the idea that a decline in type II muscle fiber SC content plays an important role in the loss of muscle mass and strength with aging.

\section{ACKNOWLEDGMENTS}

We thank all of the volunteers for their enthusiastic participation in this study.

Conflict of Interest: None of the authors had any financial or personal conflict of interest.

Author Contributions: $\mathrm{LBV}$ and $\mathrm{LJCvL}$ designed the study. LBV performed the clinical experiments, organized the data, and performed the statistical analysis. LBV and TS performed the immunohistochemical analysis and quantification. $\mathrm{MB}$ and $\mathrm{HK}$ provided medical assistance during clinical experiments. LBV, LJCvL, KM, and HHCMS were involved in interpreting the data. All authors were involved in writing the manuscript.

Sponsor's Role: The sponsor played no role in the design, methods, participant recruitment, data collections, analysis, or preparation of the paper.

\section{REFERENCES}

1. Baumgartner RN, Koehler KM, Gallagher D et al. Epidemiology of sarcopenia among the elderly in New Mexico. Am J Epidemiol 1998;147:755-763.

2. Iannuzzi-Sucich M, Prestwood KM, Kenny AM. Prevalence of sarcopenia and predictors of skeletal muscle mass in healthy, older men and women. J Gerontol A Biol Sci Med Sci 2002;57A:M772-M777.

3. Janssen I, Heymsfield SB, Ross R. Low relative skeletal muscle mass (sarcopenia) in older persons is associated with functional impairment and physical disability. J Am Geriatr Soc 2002;50:889-896. 
4. Doherty TJ. Invited review: Aging and sarcopenia. J Appl Physiol 2003;95:1717-1727.

5. Janssen I, Heymsfield SB, Wang ZM et al. Skeletal muscle mass and distribution in 468 men and women aged 18-88 yr. J Appl Physiol 2000;89:81-88.

6. Lindle RS, Metter EJ, Lynch NA et al. Age and gender comparisons of muscle strength in 654 women and men aged 20-93 yr. J Appl Physiol 1997;83:15811587.

7. Short KR, Vittone JL, Bigelow ML et al. Changes in myosin heavy chain mRNA and protein expression in human skeletal muscle with age and endurance exercise training. J Appl Physiol 2005;99:95-102.

8. Lexell J, Taylor CC, Sjostrom M. What is the cause of the ageing atrophy? Total number, size and proportion of different fiber types studied in whole vastus lateralis muscle from 15- to 83-year-old men. J Neurol Sci 1988;84: 275-294.

9. Larsson L, Grimby G, Karlsson J. Muscle strength and speed of movement in relation to age and muscle morphology. J Appl Physiol 1979;46:451-456

10. van den Beld AW, de Jong FH, Grobbee DE et al. Measures of bioavailable serum testosterone and estradiol and their relationships with muscle strength, bone density, and body composition in elderly men. J Clin Endocrinol Metab 2000;85:3276-3282.

11. Kyle UG, Genton L, Hans D et al. Total body mass, fat mass, fat-free mass, and skeletal muscle in older people: Cross-sectional differences in 60-year-old persons. J Am Geriatr Soc 2001:49:1633-1640.

12. Frontera WR, Hughes VA, Fielding RA et al. Aging of skeletal muscle: A $12-\mathrm{yr}$ longitudinal study. J Appl Physiol 2000;88:1321-1326.

13. Gallagher D, Ruts E, Visser $M$ et al. Weight stability masks sarcopenia in elderly men and women. Am J Physiol Endocrinol Metab 2000;279:E366E375.

14. Baumgartner RN, Waters DL, Gallagher D et al. Predictors of skeletal muscle mass in elderly men and women. Mech Ageing Dev 1999;107:123-136.

15. Frontera WR, Hughes VA, Lutz KJ et al. A cross-sectional study of muscle strength and mass in 45- to 78-yr-old men and women. J Appl Physiol 1991;71:644-650.

16. Visser M, Kritchevsky SB, Goodpaster BH et al. Leg muscle mass and composition in relation to lower extremity performance in men and women aged 70 to 79: The Health, Aging and Body Composition Study. J Am Geriatr Soc 2002;50:897-904.

17. Larsson L, Sjodin B, Karlsson J. Histochemical and biochemical changes in human skeletal muscle with age in sedentary males, age 22-65 years. Acta Physiol Scand 1978;103:31-39.

18. Klitgaard H, Mantoni M, Schiaffino S et al. Function, morphology and protein expression of ageing skeletal muscle: A cross-sectional study of elderly men with different training backgrounds. Acta Physiol Scand 1990;140:41-54.

19. Verdijk LB, Koopman R, Schaart G et al. Satellite cell content is specifically reduced in type II skeletal muscle fibers in the elderly. Am J Physiol Endocrinol Metab 2007;292:E151-E157.

20. Hawke TJ, Garry DJ. Myogenic satellite cells: Physiology to molecular biology. J Appl Physiol 2001;91:534-551.

21. Shefer G, Van de Mark DP, Richardson JB et al. Satellite-cell pool size does matter: Defining the myogenic potency of aging skeletal muscle. Dev Biol 2006;294:50-66.

22. Kamel HK, Maas D, Duthie EH Jr. Role of hormones in the pathogenesis and management of sarcopenia. Drugs Aging 2002;19:865-877.

23. Waters DL, Yau CL, Montoya GD et al. Serum Sex Hormones, IGF-1, and IGFBP3 exert a sexually dimorphic effect on lean body mass in aging. J Gerontol A Biol Sci Med Sci 2003;58A:648-652.

24. Bhasin S, Woodhouse L, Casaburi R et al. Older men are as responsive as young men to the anabolic effects of graded doses of testosterone on the skeletal muscle. J Clin Endocrinol Metab 2005;90:678-688.

25. Sinha-Hikim I, Cornford M, Gaytan H et al. Effects of testosterone supplementation on skeletal muscle fiber hypertrophy and satellite cells in community-dwelling older men. J Clin Endocrinol Metab 2006;91:3024-3033.
26. American Diabetes Association. Diagnosis and classification of diabetes mellitus. Diabetes Care 2006;29(Suppl):S43-S48.

27. Mackey AL, Kjaer M, Charifi $\mathrm{N}$ et al. Assessment of satellite cell number and activity status in human skeletal muscle biopsies. Muscle Nerve 2009;40:455465.

28. Mayhew JL, Prinster JL, Ware JS et al. Muscular endurance repetitions to predict bench press strength in men of different training levels. J Sports Med Phys Fitness 1995;35:108-113.

29. Verdijk LB, van Loon L, Meijer K et al. One-repetition maximum strength test represents a valid means to assess leg strength in vivo in humans. J Sports Sci 2009;27:59-68.

30. Verdijk LB, Gleeson BG, Jonkers RA et al. Skeletal muscle hypertrophy following resistance training is accompanied by a fiber type-specific increase in satellite cell content in elderly men. J Gerontol A Biol Sci Med Sci 2009;64A:332-339.

31. Mitsiopoulos N, Baumgartner RN, Heymsfield SB et al. Cadaver validation of skeletal muscle measurement by magnetic resonance imaging and computerized tomography. J Appl Physiol 1998;85:115-122.

32. Chumlea WC, Roche AF, Steinbaugh ML. Estimating stature from knee height for persons 60 to 90 years of age. J Am Geriatr Soc 1985;33:116-120.

33. Roubenoff R, Wilson PW. Advantage of knee height over height as an index of stature in expression of body composition in adults. Am J Clin Nutr 1993;57:609-613.

34. Vermeulen A, Verdonck L, Kaufman JM. A critical evaluation of simple methods for the estimation of free testosterone in serum. J Clin Endocrinol Metab 1999;84:3666-3672.

35. Bergstrom J. Percutaneous needle biopsy of skeletal muscle in physiological and clinical research. Scand J Clin Lab Invest 1975;35:609-616.

36. Hortobagyi T, Zheng D, Weidner $\mathrm{M}$ et al. The influence of aging on muscle strength and muscle fiber characteristics with special reference to eccentric strength. J Gerontol A Biol Sci Med Sci 1995;50A:B399-B406.

37. Hinkle DE, Wiersma W, Jurs SG. Applied Statistics for the Behavioral Sciences, 4th Ed. Boston, NY: Houghton Mifflin Company, 1998.

38. Fiatarone MA, Marks EC, Ryan ND et al. High-intensity strength training in nonagenarians. Effects on skeletal muscle. JAMA 1990;263:3029-3034.

39. Frontera WR, Suh D, Krivickas LS et al. Skeletal muscle fiber quality in olde men and women. Am J Physiol Cell Physiol 2000;279:C611-C618.

40. Goodpaster BH, Carlson CL, Visser M et al. Attenuation of skeletal muscle and strength in the elderly: The Health ABC Study. J Appl Physiol 2001;90: $2157-2165$.

41. Lexell J. Human aging, muscle mass, and fiber type composition. J Gerontol A Biol Sci Med Sci 1995;50A(Spec No):11-16.

42. Verney J, Kadi F, Charifi N et al. Effects of combined lower body endurance and upper body resistance training on the satellite cell pool in elderly subjects. Muscle Nerve 2008;38:1147-1154.

43. Allen DL, Roy RR, Edgerton VR. Myonuclear domains in muscle adaptation and disease. Muscle Nerve 1999;22:1350-1360.

44. Cheek DB. The control of cell mass and replication. The DNA unit-a personal 20-year study. Early Hum Dev 1985;12:211-239.

45. Kadi F, Eriksson A, Holmner S et al. Cellular adaptation of the trapezius muscle in strength-trained athletes. Histochem Cell Biol 1999;111:189195.

46. Brack AS, Bildsoe H, Hughes SM. Evidence that satellite cell decrement contributes to preferential decline in nuclear number from large fibres during murine age-related muscle atrophy. J Cell Sci 2005;118:4813-4821.

47. Allen DL, Linderman JK, Roy RR et al. Growth hormone/IGF-I and/or resistive exercise maintains myonuclear number in hindlimb unweighted muscles. J Appl Physiol 1997;83:1857-1861.

48. Alway SE, Siu PM. Nuclear apoptosis contributes to sarcopenia. Exerc Sport Sci Rev 2008;36:51-57.

49. Goldspink G. Loss of muscle strength during aging studied at the gene level. Rejuvenat Res 2007;10:397-405. 